ZOOLOGIA 27 (4): 563-568, August, 2010

doi: $10.1590 /$ S1984-46702010000400008

\title{
Redescription and new distribution records of Acanthoscurria paulensis (Araneae: Mygalomorphae: Theraphosidae)
}

\author{
Sylvia M. Lucas; Felipe dos S. Paula; Hector M. O. Gonzalez Filho \& Antonio D. Brescovit
}

Laboratório de Artrópodes, Instituto Butantan. Avenida Vital Brazil 1500, 05503-900 São Paulo, SP, Brazil. E-mail: sylvialucas@butantan.gov.br

\begin{abstract}
The male of Acanthoscurria paulensis Mello-Leitão, 1923 is redescribed and the female is described for the first time. The male holotype, from Pirassununga, state of São Paulo, Brazil, could not be located and the redescription is based on specimens collected in the type locality. Acanthoscurria atrox Vellard, 1924 is considered a junior synonym of A. paulensis based on their original descriptions and figures of the male palpal bulb, and also supported by the study of several specimens from both type localities, Pirassununga and Campo Grande, state of Mato Grosso do Sul, Brazil. Acanthoscurria guaxupe Piza, 1972 is also considered a junior synonym of $A$. paulensis based on examination of the holotype. Acanthoscurria paulensis resembles A. chacoana Brèthes, 1909, A. juruenicola Mello-Leitão, 1923, and A. geniculata (C.L. Koch, 1842) by the large size (over $50 \mathrm{~mm}$ ). The male of $A$. paulensis resembles that of $A$. chacoana, $A$. juruenicola, and $A$. geniculata by the aspect of the palpal bulb with an embolus ending like a shell, due to the well-developed prolateral inferior and superior keels. It can be distinguished from $A$. chacoana by the palpal bulb less compact and with a longer embolus and from A. juruenicola and A.geniculata by the absence of a third accessory keel. The female resembles A. chacoana, A. juruenicola, and A. geniculata by the fused base of the spermathecae, and differs by the square or slightly wider than longer base of the spermathecae and by the terminal lobes directed towards each other. New distribution records for A. paulensis are provided from the Brazilian states of Mato Grosso, Goiás, Minas Gerais, Mato Grosso do Sul, Espírito Santo, Paraná, and Rio Grande do Sul.
\end{abstract}

KEY WORDS. Acanthoscurria atrox; Acanthoscurria guaxupe; new distribution records; taxonomy; Theraphosinae.

Acanthoscurria Ausserer, 1871 currently includes 40 species, two from Central America and 38 from South America (Platnick 2010). Twenty-seven South American species are cited for or described from Brazil (PLATnick 2010). Only 12 species are known from both sexes. Unfortunately, most descriptions date from the XIX and first half of the XX century (Косн 1841, CAmbridge 1896) and do not mention characters such as the aspect of spermathecae and palpal bulb morphology, which are considered important taxonomic traits nowadays. Several type specimens are pinned in dry collections and are too fragile to examine. Many other type specimens could not be located and are considered lost.

Ten Acanthoscurria species were described from Brazil by Mello Leitão (1923), including A. paulensis, based on a male from Pirassununga, state of São Paulo. He did not mention the morphology of the male palpal bulb and seminal receptacle, which are important for the identification of species. The spider collection of the Instituto Butantan holds a great number of specimens belonging to this genus and several from the type locality. This material enabled us to detect the conspecific female and to describe and illustrate male and female sexual structures. In ad- dition, we establish the synonymies of $A$. atrox Vellard, 1924 and A. guaxupe Piza, 1972 with $A$. paulensis. The distribution range of $A$. paulensis is extended to other Brazilian states.

\section{MATERIAL AND METHODS}

The material examined is deposited in the collection of the Instituto Butantan, São Paulo (IBSP, curator: I. Knysak). Spine notation follows Petrunkevitch (1925). All measurements are in millimeters. Female spermathecae were dissected and cleared in lactic acid for observation of internal structures. The length of leg segments was measured between joints in dorsal view. Length and width of carapace, eye tubercle, labium, and sternum represent maximum values. Total body length excludes pedicel and spinnerets. The drawings were made on a Leica MZ 12 stereoscope with the aid of a camera lucida. Abbreviations: (AME) anterior median eyes, (ALE) anterior lateral eyes, (PLE) posterior lateral eyes, (PME) posterior median eyes, (STC) superior tarsal claws; spines: (ap) apical, (d) dorsal, (v) ventral, (p) prolateral, (r) retrolateral; bulb keels: (PI) prolateral inferior, (PS) prolateral superior, (A) apical (BERTANI 2000). 


\section{TAXONOMY}

\section{Acanthoscurria paulensis Mello Leitão, 1923}

Figs 1-9

Acanthoscurria paulensis Mello Leitão, 1923: 304, figs 86-88, 178179 (holotype male from Pirassununga, state of São Paulo, should be in the Museu Nacional, Universidade Federal do Rio de Janeiro, not located, considered lost by the curator, A.B. Kury); Platnick, 2010.

Acanthoscurria atrox Vellard, 1924: 143, figs 35a-f (holotype male and paratype female from Campo Grande, state of Mato Grosso do Sul, should be in the Instituto Vital Brazil, Niterói, considered lost by the curator, C.M.V. Souza); Bücherl, 1957: 396, figs 58, 58a-b; Bertani, 2000: 30, figs 25-26; Platnick, 2010. Syn. nov.

Acanthoscurria guaxupe Piza, 1972: 161, fig. 1 (holotype male from Guaxupé, state of Minas Gerais, deposited in the Instituto Butantan, São Paulo, IBSP 138247, examined); Platnick, 2010. Syn. nov.

Diagnosis. Acanthoscurria paulensis resembles A. chacoana, A. juruenicola, and A. geniculata by the large body size (over 50 $\mathrm{mm}$ in total length), by the aspect of the male palpal bulb with an embolus ending like a shell, due to the well developed prolateral inferior and superior keels (Mello-Leitão 1923, fig. 178), and by the female spermathecae with a fused base (Schiapelli \& Gerschman de Pikelin 1964, plate III, fig. 3, Bertani \& DA SILVA 2004, figs 9-12). Acanthoscurria paulensis can be distinguished from the other species of the genus by the palpal bulb less compact and with a longer embolus and by the absence of a third accessory keel (Figs 1-3) and by the more square or slightly wider than longer base of the spermathecae with terminal lobes directed towards each other (Figs 7 and 8).

Description. Male (IBSP 107454). Coloration in live: carapace dark brown bordered with reddish hairs; chelicerae black covered with long reddish hairs. Labium and sternum black. Leg coxae and femora black, other segments brown, with two longitudinal stripes on patellae, tibiae and metatarsi. Abdomen black, dorsal side covered with long reddish hairs. Total length 60.5. Carapace 25.0 long, 21.0 wide. Fovea procurved. Clypeus narrow. Eye group rectangular. Anterior eye row procurved, posterior recurved. Eye sizes: AME 0.55, ALE 0.70, PLE 0.70, PME 0.30. Eye tubercle 2.5 long, 3.6 wide. Labium 3.5 long, 3.0 wide, with 110 cuspules. Endites with 282 cuspules each. Sternum moderately convex, 13.0 long, 10.0 wide, with posterior sigillae three times larger than anterior pair. Cheliceral furrow with 10 teeth and with 32 smaller basal teeth. Stridulatory apparatus with around 20 bristles. STC I with 8 teeth, II with 9 , III with and IV with 8. Palp: femur length 13.0, patella 7.0, tibia 11.0, cymbium 6.5, total 37.5. Legs: I: femur 18.5, patella 10.0, tibia 15.0, metatarsus 15.0, tarsus 11.0, total 69.5; II: 19.0, 9.5, 13.5, 14.5, 10.0, 66.5; III: 16.5, 9.0, 12.0, 16.0, 9.5, 63.0; IV: $20.0,9.5,15.0,20.0,10.5,75.0$. Spines: palp: femur d0-0-0- 1ap, patella p0-1-0, tibia p0-1-0-0-0-0-1-1-0-0-2-3, r0-0-0-1; I: femur d0-0-0-1p, patella v0-0-1, tibia v0-3-0-2-0-1p-2-1r-1p-21r-1p-6ap, metatarsus v0-0-3ap; II: femur p0-0-1, patella v0-02ap, tibia v0-2-0-1-1-0-1-2-0-1p-1-1r-0-6ap, metatarsus v1r-01-1r-0-0-1ap; III: femur d0-0-0-0-1p-1r, patella p0-1-0, r1-0-0, tibia v0-1r-0-1-2p-0-4-1p-1p-1r-1p-0-5ap, metatarsus v0-2p-1p1-1-1p-1r-1r-1p-1r-0-0-1r-1p-4ap; IV: femur d0-0-0-1r, tibia d20-2-0, v0-0-0-2r-0-0-1r-3ap, metatarsus d0-0-0-2, v1p-0-1p-01p-0-1p-1p-2p-1r-0-0-1r-1r-0-2r-1r-1p-1r-6ap. Scopulae on metatarsi I-II present throughout ventral portion, restricted to apical half of metatarsi III and apical third of IV. All tarsi with scopulae. Tibial apophysis of leg I with 8-15 spines on apex (Figs 4 and 5). Palpal tibia with blunt tubercle (Fig. 6). Male palpal bulb with long and thickened embolus ending in shell formed by well developed superior and inferior keels and small one next to opening (Figs 1-3).

Female (IBSP 107776). Coloration as in male (Fig. 9). Total length 68.5. Carapace 26.5 long, 22.0 wide. Fovea procurved. Clypeus narrow. Eye group rectangular. Anterior eye row procurved, posterior recurved. Eye sizes: AME 0.6, ALE 0.6, PLE 0.7, PME 0.3. Eye tubercle 2.6 long, 3.7 wide. Labium 4.0 long, 3.0 wide, with 141 cuspules. Endites with 318 cuspules each. Sternum moderately convex, 12.0 long, 10.5 wide, with posterior sigillae three times larger than anterior pair. Cheliceral furrow with 14 teeth and 51 smaller basal teeth. Stridulatory apparatus with around 20 bristles. STC I-II-III with 5-6 teeth and IV with 7 teeth. Palp: femur length 14.5 , patella 5.5, tibia 10.0, cymbium 10.0, total 40.0. Legs: I: femur 17.5, patella 11.0, tibia 15.0, metatarsus 13.0, tarsus 7.0, total 63.5. II: 16.0, 10.0, 12.0, 12.0, 8.0, 58.0. III: $14.5,8.5,11.0,13.0,7.0,54.0$. IV: 17.0, 10.0, 13.0, 18.0, 9.0, 67.0. Spines: palp: femur d0-0-0-1p, patella d0-1p-1p-0-0-0, tibia v0-1p-0-1r-2p-0-0-5ap. I: femur d00-0-0-0-0-0-0-1p-1p-0, tibia v0-1-0-0-2-1r-1p-0-0-3ap, metatarsus v0-1r-0-0-0-3ap. II: femur d0-0-2-1p, patella d0-1p-0, tibia v0-1r-0-0-2-1r-0-0-3ap, metatarsus v0-1r-0-0-0-3ap. III: femur d0-1r-0-1r, patella d0-1r-0-0, tibia v0-1r-0-2-1r-1p-0-2ap, metatarsus v2p-0-1-1p-1r-1p-1r-0-0-1r-1p-5ap. IV: femur d0-0-0-1r, tibia v0-1r-0-0-2-0-1r-0-0-3ap, metatarsus v2-0-2-2r-1p-2r-1p1r-3-0-1p-1r-2r-1r-1p-1r-1r-1p-1p-4ap. Scopulae on metatarsi III present throughout ventral portion and restricted to apical third in metatarsi III-IV. All tarsi with scopulae. Spermathecae with large base, slightly larger than long (Figs 7 and 8).

Material examined. Brasil, Mato Grosso: São Felix do Araguaia, $11^{\circ} 37^{\prime} 01^{\prime \prime S}, 50^{\circ} 40^{\prime} 08^{\prime \prime} \mathrm{W}, 1$ female, XII.1974, I.

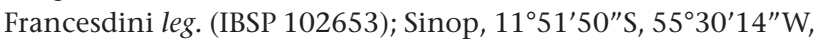
1 female, V.1984, A. Pazzim Filho leg. (IBSP 107110); Canarana, $13^{\circ} 33^{\prime} 00^{\prime \prime}$, 52 ${ }^{\circ} 9^{\prime} 57^{\prime \prime} \mathrm{W}, 1$ male, 16.XII.2000-10.I.2001, R.A.K. Ribeiro \& C.A. Falcetti leg. (IBSP 112138); Chapada dos Guimarães, Rio Manso, Furnas, $1^{\circ} 27^{\prime} 39^{\prime \prime}$, 5 5 $5^{\circ} 45^{\prime} 00^{\prime \prime} \mathrm{W}, 1 \mathrm{fe}-$ male, 2000, Equipe Resgate Fauna leg. (IBSP 109046); Cuiabá, $15^{\circ} 35^{\prime} 45^{\prime \prime} \mathrm{S}, 56^{\circ} 05^{\prime} 49^{\prime \prime} \mathrm{W}, 1$ male, 20.VIII.1987, R. Veloso leg. (IBSP 110726); Cáceres, $16^{\circ} 04^{\prime} 16^{\prime \prime} \mathrm{S}, 57^{\circ} 40^{\prime} 44^{\prime \prime} \mathrm{W}, 1$ male (IBSP 103694); Poconé, 1 female, XII.1989, R. Bertani leg. (IBSP 


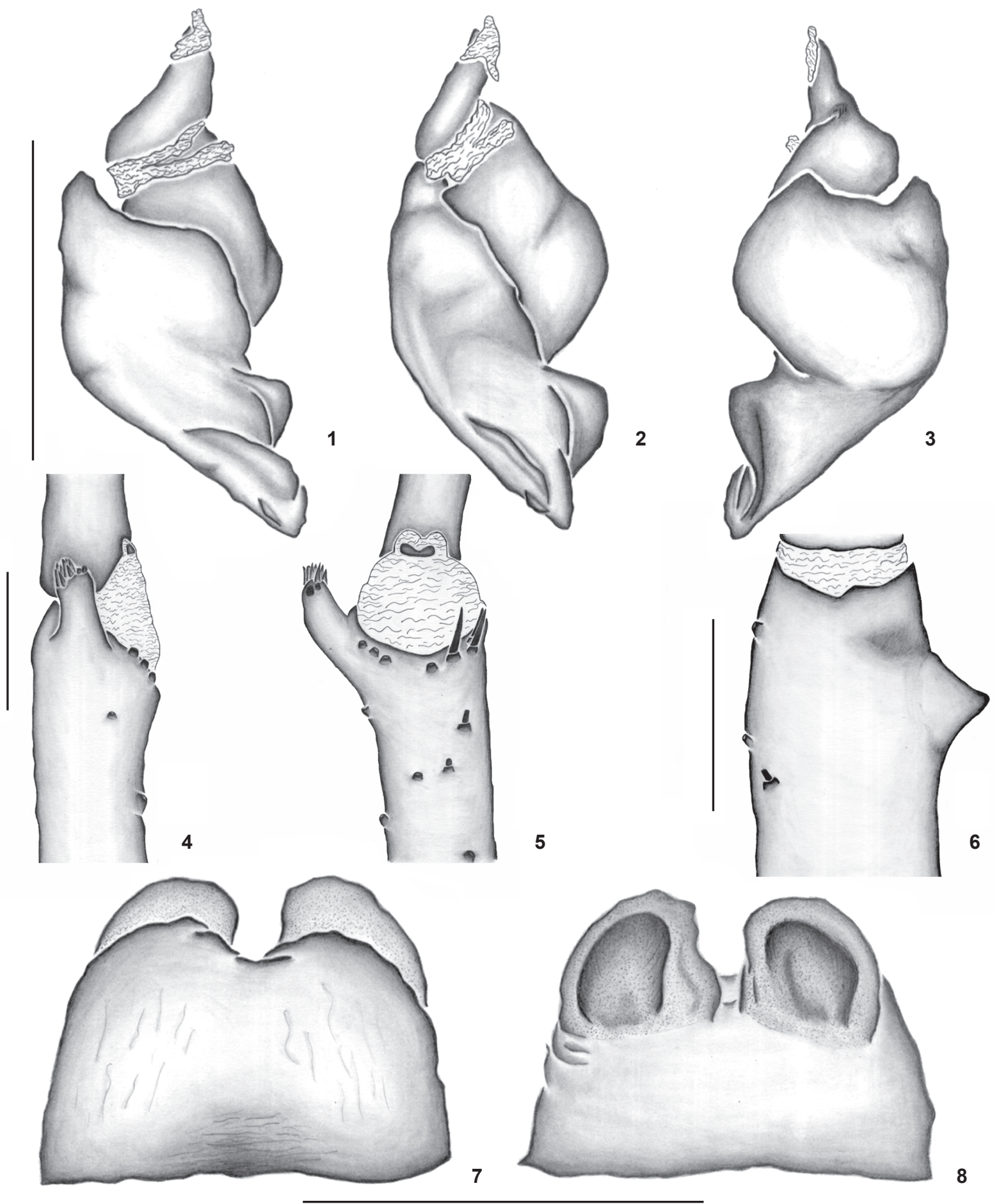

Figures 1-8. Acanthoscurria paulensis. (1-3) Left male palpal bulb, IBSP 107454: (1) prolateral view; (2) prolateral-ventral view; (3) retrolateral view; (4-5) left tibial apophysis; (6) left palpal tibia; (7-8) female spermathecae, IBSP 107776: (7) dorsal view; (8) ventral view. Scale bars $=5 \mathrm{~mm}$. 


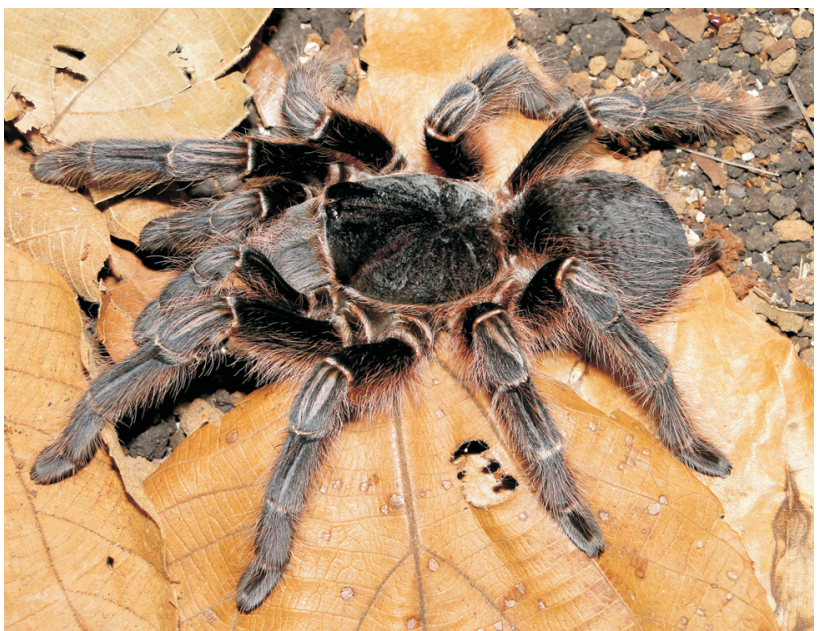

Figure 9. Acanthoscurria paulensis, female from Itupeva, state of São Paulo, dorsal view. Photo: R.P. Indicatti.

107516); Itiquira, Fazenda das Cachoeiras, $17^{\circ} 12^{\prime} 32^{\prime \prime} \mathrm{S}$, 5409'00”W, 1 female, VIII.1985, (IBSP 104879). Goiás: Minaçu, Usina Hidrelétrica da Serra da Mesa, 1331'37"S, 48¹3'14"W, 1 female, 1996, Resgate de Fauna leg. (IBSP 111937); Crixás, Fazenda Tarumã, $14^{\circ} 32^{\prime} 56^{\prime \prime}$ S, 49 5 $^{\prime} 8^{\prime} 08^{\prime \prime} \mathrm{W}, 2$ males, 25.II.1976, V. B. Buchaul leg. (IBSP 104178); Mozarlandia, 1444'26"S, $50^{\circ} 33^{\prime} 07^{\prime \prime} \mathrm{W}, 1$ female, 6.VIII.1976, W.M. Sposito leg. (IBSP 104207); Aruanã, $14^{\circ} 55^{\prime} 12^{\prime \prime} \mathrm{S}, 5^{\circ} 04^{\prime} 58^{\prime \prime} \mathrm{W}, 1$ male, II.1990, E.

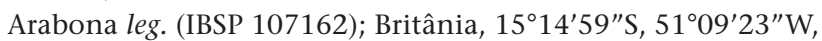
1 male, IV.1991, J.R.C. Stamato leg. (IBSP 107493); Jussara, $15^{\circ} 51^{\prime} 54^{\prime \prime} \mathrm{S}, 50^{\circ} 52^{\prime} 04^{\prime \prime} \mathrm{W}, 1$ male, 1985, A. Lima leg. (IBSP 107077); Corumbá de Goiás, $15^{\circ} 55^{\prime} 27^{\prime \prime S}, 48^{\circ} 48^{\prime} 31^{\prime \prime} \mathrm{W}, 6$ females, 03.VI.1986, E. Snartele leg. (IBSP 104918A); Goiás, $15^{\circ} 55^{\prime} 20^{\prime \prime} \mathrm{S}, 50^{\circ} 08^{\prime} 16^{\prime \prime} \mathrm{W}, 1$ male, II.1990, M. Caxibona leg. (IBSP 107167); Aragarças, $15^{\circ} 53^{\prime} 52^{\prime \prime} \mathrm{S}, 52^{\circ} 15^{\prime} 03^{\prime \prime} \mathrm{W}, 1$ female, 25.IV.1969, R. Viana leg. (IBSP 103917); Luiziânia, Usina Hidrelétrica de Corumbá, $16^{\circ} 20^{\prime} 47^{\prime \prime} S-48^{\circ} 10^{\prime} 44^{\prime \prime} \mathrm{W}, 1$ female, 22.XI.2005, Equipe Butantan leg. (IBSP 112936); Inhumas, $16^{\circ} 21^{\prime} 28^{\prime \prime}$, $49^{\circ} 29^{\prime} 45^{\prime \prime} \mathrm{W}, 1$ female, 7.I.1970, E.E. Bergold leg. (IBSP 104027); Goiana, Jardim Goiás, $16^{\circ} 40^{\prime} 00^{\prime \prime} S$, 49 $9^{\circ} 15^{\prime} 00^{\prime \prime} \mathrm{W}$, 1 female, 02.II.2002, Consultoria Ambiental leg. (IBSP 113443); Catalão, Cônego São Domingos, 18¹0'12"S, 4756'31"W, 1 male, 19-22.IX.1999, Escola Arminda Rosa de Mesquita leg. (IBSP 107990); Chapadão do Céu, 18²3'34"S, 52³9'57"W, 1 female, 11.IV.01, C. Pmtsch leg. (IBSP 109128). Minas Gerais: Mato Verde, $15^{\circ} 23^{\prime} 49^{\prime \prime} \mathrm{S}, 42^{\circ} 51^{\prime} 57^{\prime \prime} \mathrm{W}, 1$ female, 27.IX.1948, Equipe IBSP leg.

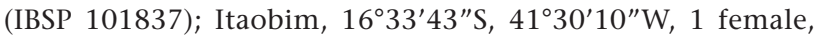
12.XI.1952, J.Purini leg. (IBSP 100192); Paracatu, 17²13'19"S, $46^{\circ} 52^{\prime} 30^{\prime \prime} \mathrm{W}, 1$ male, II.1983, Coop. Agrícola de Cotia leg. (IBSP 104755); Ibiaí, $16^{\circ} 51^{\prime} 39^{\prime \prime}$ S, 4454'50”W, 1 male, III.1989, A.E.O. Paciullo leg. (IBSP 107090); Várzea da Palma, 17³5'52"S, $44^{\circ} 43^{\prime} 51^{\prime \prime} \mathrm{W}, 1$ male, 20.III.1978, Eletrometalur leg. (IBSP 104316); Pirapora, $17^{\circ} 20^{\prime} 42^{\prime \prime}$ S, $44^{\circ} 56^{\prime} 31^{\prime \prime} \mathrm{W}, 1$ male, 11.II.2000

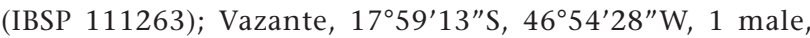
IX.1985 (IBSP 104877); Três Marias, $18^{\circ} 12^{\prime} 21^{\prime \prime}$ S, 45¹4'31”W, 1 male, 15.XII.1977, Cia. Reflorestamento Rio Escuro leg. (IBSP 104287); Patos de Minas, $18^{\circ} 34^{\prime} 44^{\prime \prime} \mathrm{S}, 46^{\circ} 31^{\prime} 04^{\prime \prime} \mathrm{W}, 1$ female, VII.2002, K. Rubia leg. (IBSP 111591); Cascalho Rico, 18³4'33"S, 47²5'37"W, 1 male, IX.1982, Serraria Guarani Ltda. leg. (IBSP 107078); Araguari, $18^{\circ} 38^{\prime} 56^{\prime \prime} \mathrm{S}, 48^{\circ} 11^{\prime} 13^{\prime \prime} \mathrm{W}, 1$ female, X.1986, C. R. Marques leg. (IBSP 107270); Uberlândia, 1855'08"S, $48^{\circ} 16^{\prime} 37^{\prime \prime} \mathrm{W}, 1$ female, 8.III.1954, B. Carvalho leg. (IBSP 103187); Indianópolis, $19^{\circ} 02^{\prime} 20^{\prime \prime} \mathrm{S}, 47^{\circ} 55^{\prime} 01^{\prime \prime} \mathrm{W}, 1$ male, IX.1986, C. Dall'Aglio leg. (IBSP 107720); Prata, 19²18'25"S, 4855'26"W, 1 female, IV.2001, Prefeitura Municipal leg. (IBSP 108802); Gurinhatã, $19^{\circ} 12^{\prime} 00^{\prime \prime}$ S, $49^{\circ} 46^{\prime} 00^{\prime \prime} \mathrm{W}, 1$ male, VI.1989, J.D.S. Souza leg. (IBSP 107512); Sacramento, $19^{\circ} 51^{\prime} 54^{\prime \prime}$ S, 47 $26^{\circ} 24^{\prime \prime} \mathrm{W}$, 1 male, 24.III.1977, Seminário Santíssimo Redenta leg. (IBSP 104247); Belo Horizonte, $19^{\circ} 49^{\prime} 01^{\prime \prime} \mathrm{S}, 43^{\circ} 57^{\prime} 21^{\prime \prime} \mathrm{W}, 1$ male, 22.III.1977, N. de S. Lima leg. (IBSP 104243); Santo Antônio do Monte, $20^{\circ} 05^{\prime} 13^{\prime \prime}$ S, 4537'38”W, 1 female, IV.1991, P.R.F. Espíndola leg. (IBSP 107503); Alpinópolis, 2051’50"S, 46²3'16"W, 1 male, II.1983, J. Oliveira leg. (IBSP 104759); Barbacena, $21^{\circ} 13^{\prime} 33^{\prime \prime} \mathrm{S}, 43^{\circ} 46^{\prime} 26^{\prime \prime} \mathrm{W}, 1$ female, 13.II.1948, K.

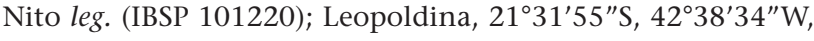
1 female, VII.1953, W.M. Silva leg. (IBSP 102475). Distrito Federal: Brasília, $15^{\circ} 46^{\prime} 48^{\prime \prime}$ S, 4755’45”W, 1 female, X.1989, P. S. Sumodigo leg. (IBSP 107481). Mato Grosso do Sul: Corumbá, $19^{\circ} 0^{\prime} 32^{\prime \prime}$ S, 57 39'10"W, Passo do Lontra, 1 female, I.1999, J. Raizer leg. (IBSP 110059); Coxim, 18 $30^{\circ} 25^{\prime \prime}$, 54 $4^{\circ} 45^{\prime} 36^{\prime \prime} \mathrm{W}, 2$ males, 12.III.1976, O.G. de Moraes leg. (IBSP 104183); Aquidauana, $20^{\circ} 28^{\prime} 15^{\prime \prime} \mathrm{S}, 5^{\circ} 47^{\prime} 13^{\prime \prime} \mathrm{W}, 1$ male, C. Roloff leg. (IBSP 107094); Paranaíba, 1940’37"S, 51¹1'27”W, 1 male, XI.1986, R.R. Silva leg. (IBSP 107353); Aparecida do Taboado, $20^{\circ} 05^{\prime} 13^{\prime \prime S}, 51^{\circ} 05^{\prime} 38^{\prime \prime} \mathrm{W}, 1$ female, XI.1982, E. Marin leg. (IBSP 104736); Miranda, 20¹4'27"S, 56²2'40"W, 1 female, 24.IX.1952, C. Moraes leg. (IBSP 103027); Campo Grande, $20^{\circ} 26^{\prime} 34^{\prime \prime}$ S, 54 $38^{\circ} 45^{\prime \prime} \mathrm{W}, 1$ male, 3.VIII.1979, J.M. Midorikawa

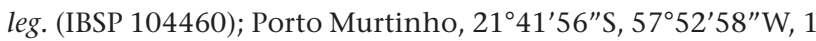
male, 3.V.2004, (IBSP 12094); Brasilândia, 2115'21"S, $52^{\circ} 02^{\prime} 13^{\prime \prime} \mathrm{W}, 2$ males, 2006, Prefeitura Municipal leg. (IBSP 113060); Bonito, $21^{\circ} 07^{\prime} 15^{\prime \prime} \mathrm{S}, 56^{\circ} 28^{\prime} 55^{\prime \prime} \mathrm{W}, 1$ female, 09.XII. 1994, G. Christianini \& F. Peixoto leg. (IBSP 107975); Rio Brilhante, $21^{\circ} 48^{\prime} 07^{\prime \prime S}, 54^{\circ} 32^{\prime} 45^{\prime \prime} \mathrm{W}, 2$ males, 1 immature, 3.III.1969, M.C. Pernambucanas leg. (IBSP 103897); Nova Andradina, $22^{\circ} 13^{\prime} 58^{\prime \prime}$ S, $53^{\circ} 20^{\prime} 34^{\prime \prime} \mathrm{W}, 1$ female, XII.1985, C.A. Cardoso leg. (IBSP 104902). Espírito Santo: Itapemirim, $21^{\circ} 00^{\prime} 40^{\prime \prime} \mathrm{S}, 40^{\circ} 50^{\prime} 02^{\prime \prime} \mathrm{W}, 1$ male, II.1996, R. Cassaninga leg. (IBSP 107582). São Paulo: Guarani do Oeste, 2004'30"S, $50^{\circ} 20^{\prime} 20^{\prime \prime} \mathrm{W}, 1$ female, 1978, G. Puorto leg. (IBSP 107305); Barretos, Palmar, 20³3'26"S, 48 34'04"W, 2 females, 7.V.1947, L. Bergantini leg. (IBSP 100330); Morro Agudo, 2043'51"S, $48^{\circ} 03^{\prime} 28^{\prime \prime} \mathrm{W}, 1$ male, 20.V.1986, P.B. Camargo leg. (IBSP 104917); Colina, E.F. Palmar, $20^{\circ} 42^{\prime} 49^{\prime \prime}$ S, 48 $32^{\prime} 28^{\prime \prime}$ W, 1 female, 8.IV.1947, L. Bergantini leg. (IBSP 100229); Altinópolis, $21^{\circ} 01^{\prime} 23^{\prime \prime} \mathrm{S}, 47^{\circ} 22^{\prime} 22^{\prime \prime} \mathrm{W}, 1$ female, 26.VII.1947, Shubat leg. (IBSP 
100594); Pontal, $21^{\circ} 01^{\prime} 22^{\prime \prime} \mathrm{S}, 48^{\circ} 02^{\prime} 13^{\prime \prime} \mathrm{W}, 1$ male, 7.II.1951, A. Romero leg. (IBSP 102466); Guararapes, 21¹5'39"S, 50³8' $34^{\prime \prime} \mathrm{W}, 1$ female, 3.XII.1969, Prefeitura Municipal leg. (IBSP 104169); Ribeirão Preto, $21^{\circ} 10^{\prime} 40^{\prime \prime} \mathrm{S}, 47^{\circ} 48^{\prime} 36^{\prime \prime} \mathrm{W}, 1$ male, 1 female, 18.VI.1975, Casa da Cultura leg. (IBSP 104143); Cajuru, $21^{\circ} 16^{\prime} 31^{\prime \prime} \mathrm{S}, 47^{\circ} 18^{\prime} 15^{\prime \prime} \mathrm{W}, 1$ male, II.1990, J.B.B. Giglio leg. (IBSP 107161); Araraquara, $21^{\circ} 47^{\prime} 55^{\prime \prime} \mathrm{S}, 48^{\circ} 11^{\prime} 15^{\prime \prime} \mathrm{W}, 1$ male, III.1993, Centro de Tratamento Regional de Acidentes leg. (IBSP 107306); Casa Branca, $21^{\circ} 46^{\prime} 26^{\prime \prime} \mathrm{S}, 47^{\circ} 5^{\prime} 9^{\prime \prime} \mathrm{W}, 1$ male, 14.V.1975, P.M. Bortolotti leg. (IBSP 104124); São Carlos, 22 01'4"S, 4753'27"W, 1 female, IV.1987, J.C. Oliveira leg. (IBSP 107615); Descalvado, $21^{\circ} 54^{\prime} 14^{\prime \prime} \mathrm{S}, 47^{\circ} 36^{\prime} 10^{\prime \prime} \mathrm{W}, 1$ female, Mineração Jundu S.A. leg. (IBSP 104845); Martinópolis, $22^{\circ} 08^{\prime} 45^{\prime \prime} \mathrm{S}, 51^{\circ} 10^{\prime} 15^{\prime \prime} \mathrm{W}, 1$ female, 31.X.1975, H.T. Ubele leg. (IBSP 104163); Pirassununga, $21^{\circ} 59^{\prime} 45^{\prime \prime} \mathrm{S}, 47^{\circ} 25^{\prime} 33^{\prime \prime} \mathrm{W}, 5$ males 6 females, 3/II/1969-15/V/ 1975, J. Moino et al. leg. (IBSP 103883, 103937, 104051, 113852, 104137); Pirassununga, $21^{\circ} 59^{\prime} 45^{\prime \prime} \mathrm{S}, 47^{\circ} 25^{\prime} 33^{\prime \prime} \mathrm{W}, 1$ male, 2 females, XI.1985-IX.1990, A.L. Prado et al. leg. (IBSP 107454, 107258, 107776); Quatá, $22^{\circ} 14^{\prime} 52^{\prime \prime} \mathrm{S}, 50^{\circ} 41^{\prime} 52^{\prime \prime} \mathrm{W}, 1$ female, 1 immature, 25.V.1948, Farmácia Paulista leg. (IBSP 101492); $22^{\circ} 21^{\prime} 07^{\prime \prime S}, 48^{\circ} 46^{\prime} 30^{\prime \prime} \mathrm{W}$, Pederneiras, 1 female, 12.VI.1947, E.

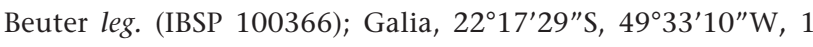
male, 19.II.1952, Z. Bonfim leg. (IBSP 102802); Mogi Mirim, $22^{\circ} 25^{\prime} 55^{\prime \prime}$, $46^{\circ} 57^{\prime} 28^{\prime \prime} \mathrm{W}, 1$ female, VII.1995, F.Q. Figueiredo leg. (IBSP 107651); Campinas, 22 $54^{\prime} 03.29^{\prime \prime} \mathrm{S}, 47^{\circ} 03^{\prime} 26.26^{\prime \prime} \mathrm{W}$, 1 female, 21.X.1949, A.J.B. Oliveira leg. (IBSP 102029); Itupeva, $23^{\circ} 09^{\prime} 10^{\prime \prime} \mathrm{S}, 47^{\circ} 03^{\prime} 28^{\prime \prime} \mathrm{W}, 1$ male, 19.II.2003, I. Madeira leg. (IBSP 110547); Barueri, $23^{\circ} 30^{\prime} 40^{\prime \prime} \mathrm{S}, 46^{\circ} 52^{\prime} 35^{\prime \prime} \mathrm{W}, 1$ female, III.1985, P. Kanematu leg. (IBSP 104856); Jundiaí, 2311'09"S, $46^{\circ} 53^{\prime} 02^{\prime \prime} \mathrm{W}, 1$ female, IV.1987, Rede Ferroviária Federal leg. (IBSP 107083); Itú, 2315'50"S, 47²17'56"W, 1 female, VI.1989, A.V. Pesek leg. (IBSP 107091); Boituva, Fazenda São Bernardo, $23^{\circ} 17^{\prime} 00^{\prime \prime S}, 47^{\circ} 40^{\prime} 20^{\prime \prime} \mathrm{W}, 1$ female, I.1991, A. Campos leg. (IBSP 104199); Sorocaba, $23^{\circ} 30^{\prime} 07^{\prime \prime} S, 47^{\circ} 27^{\prime} 28^{\prime \prime} \mathrm{W}, 1$ male, 5.III.1975, J.C. Carvalho leg. (IBSP 104120A); Capela do Alto, 23²8'14"S, $47^{\circ} 44^{\prime} 05^{\prime \prime} \mathrm{W}, 1$ female, 13.XI.1979, A.R. Queiroz leg. (IBSP 104497A); Itapetininga, $23^{\circ} 35^{\prime} 31^{\prime \prime} \mathrm{S}, 48^{\circ} 03^{\prime} 10^{\prime \prime} \mathrm{W}, 1$ male, III.1981, N. Onoda leg. (IBSP 104626A). Paraná: Palmeiras, $25^{\circ} 25^{\prime} 44^{\prime \prime} \mathrm{S}, 50^{\circ} 00^{\prime} 21^{\prime \prime} \mathrm{W}, 1$ male, 11.IV.1947, (IBSP 100278); Araucária, $25^{\circ} 35^{\prime} 34^{\prime \prime} \mathrm{S}, 4^{\circ} 24^{\prime} 36^{\prime \prime} \mathrm{W}, 1$ male, 6.II.1948, J. Silva leg. (IBSP 101213). Rio Grande do Sul: Santa Cruz do Sul, $29^{\circ} 43^{\prime} 04^{\prime \prime}$ S, 52²5'33”W, 1 male, 17.X.1950, R. Steinhaus leg. (IBSP 102316).

Distribution. Brazil: states of Mato Grosso, Goiás, Minas Gerais, Mato Grosso do Sul, São Paulo, Paraná, and Rio Grande do Sul (Fig. 10).

Remarks. the description of Mello Leitão (1923) of $A$. paulensis and that of Vellard (1924) of A. atrox are detailed and both agree in general aspect, color and measurements. The morphology of the male palpal bulb is the same in both descriptions, which allowed us to propose the synonymy. The examination of specimens from the two type localities, Pirassununga (São Paulo) and Campo Grande (Mato Grosso do

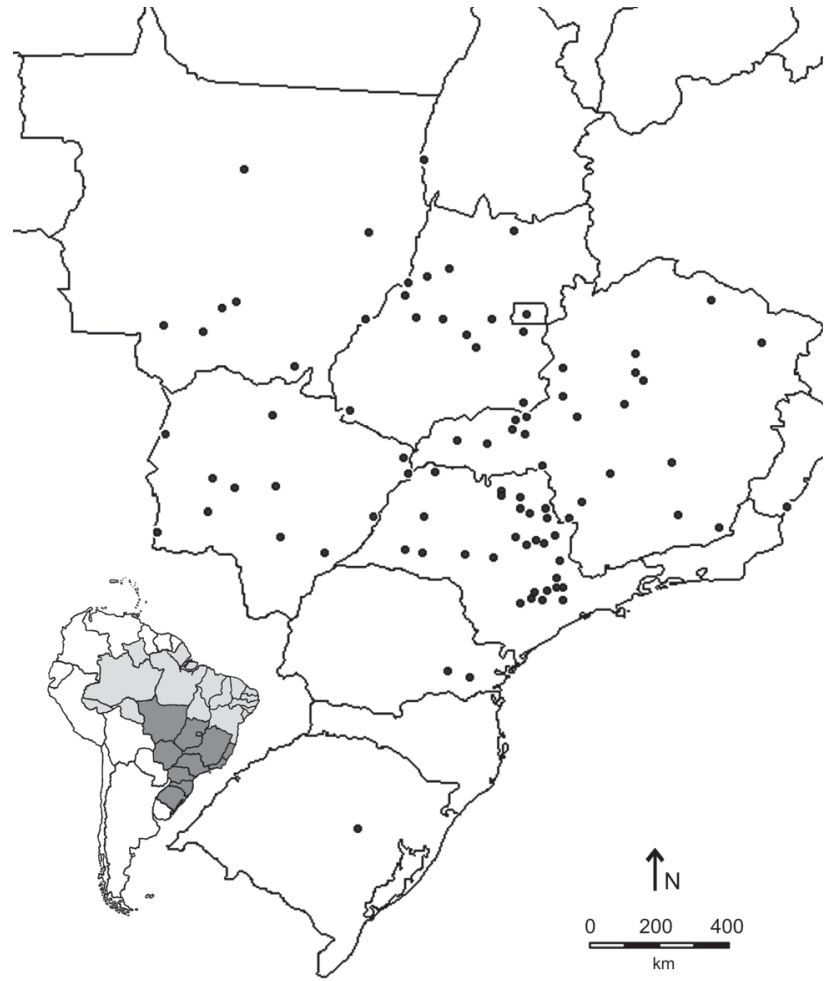

Figure 10. Known distribution of Acanthoscurria paulensis.

Sul), corroborates the synonymy. Additionaly, the examination of the holotype of A. guaxupe confirmed the synonymy with $A$. paulensis. The distinguishing character cited by PIzA (1972), number of spines on tibial apophysis of leg I, varies between eight and fourteen and the measurements of carapace and tibia plus patella of leg I and IV is the same in both species.

\section{ACKNOWLEDGEMENTS}

We wish to thank Cristina Rheims, Flávio U. Yamamoto, and Rafael P. Indicatti for helpful comments on the manuscript and assistance. Also, we wish to thank Adriano Kury, curator of the Museu Nacional, Universidade Federal do Rio de Janeiro, and Cláudio M.V. Souza, curator of the Instituto Vital Brazil, Niterói, for information about the types and the two referees for their comments on the manuscript. This work was supported by funds from the INCTTOX PROGRAM of Conselho Nacional de Desenvolvimento Científico e Tecnológico to FSP (ATP) and $\mathrm{ADB}(\mathrm{PQ})$ and Fundação de Amparo à Pesquisa do Estado de São Paulo.

\section{LITERATURE CITED}

BERTANI, R. 2000. Male palpal bulbs and homologous features in Theraphosinae (Araneae, Theraphosidae). Journal of Arachnology 28: 29-42. 
Bertani, R. \& S.C. DA Silva. 2004. Notes on Acanthoscurria chacoana Bréthes, 1909 (Araneae: Theraphosidae) in Brazil, and its synonymy with Acanthoscurria altimanni Schimdt, 2003. Zootaxa 648: 1-8.

BÜCHERL, W. 1957. Sobre a importância dos bulbos copuladores e das apófises tibiais dos machos na sistemática das aranhas caranguejeiras (Orthognatha). Anais da Academia Brasileira de Ciências 29 (3): 377-416.

CAmbridge, F.O.P. 1896. On the Theraphosidae of the Lower Amazons: being an Account of the new Genera and species of this group of Spiders discovered during the Expedition of the Steamship "Faraday" up the river Amazons. Proceedings of the Zoological Society of London 1896: 716-766.

Косн, C.L. 1841. Die Arachniden. Nürnberg, Achter Band, p. 41-131, Neunter Band, p. 1-56.

Mello-Leitão, C.F. DE. 1923. Theraphosoideas do Brasil. Revista do Museu Paulista 13: 1-438.

Petrunkevitch, A. 1925. Arachnida from Panama. Transactions of the Connecticut Academy of Arts and Sciences 27: 51248.

PIZA JR, S. DE T. 1972. Uma nova aranha Theraphosidae do Brasil. Revista de Agricultura 47: 161-162.

Platnick, N.I. 2010. The world spider catalog, version 9.5. American Museum of Natural History. Available online at: http://research.amnh.org/entomology/spiders/catalog/ intro1.html [Accessed: 08/I/2010].

Schiapelli, R.D. \& B.S. Gerschman de Pikelin. 1964. El genero Acanthoscurria Ausserer 1871 (Araneae: Theraphosidae) en la Argentina. Physis 24: 391-417.

Vellard, J. 1924. Études de Zoologie. II Sous Ordre des Mygalomorphae. Archivos do Instituto Vital Brazil 2 (2): 121-170.

Submitted: 05.I.2010; Accepted: 30.V.2010.

Editorial responsibility: Gabriel L.F. Mejdalani 